\title{
A conceptual framework for invasion in microbial communities
}

\author{
Marta Kinnunen ${ }^{1}$, Arnaud Dechesne ${ }^{1}$, Caitlin Proctor ${ }^{2}$, Frederik Hammes ${ }^{2}$, David Johnson ${ }^{2}$, \\ Marcos Quintela-Baluja ${ }^{3}$, David Graham ${ }^{3}$, Daniele Daffonchio ${ }^{4}$, Stilianos Fodelianakis ${ }^{4}$, \\ Nicole Hahn ${ }^{5}$, Nico Boon ${ }^{5}$ and Barth F Smets ${ }^{1}$ \\ ${ }^{1}$ Department of Environmental Engineering, Technical University of Denmark, Kgs. Lyngby, Denmark; ${ }^{2}$ Eawag, \\ Swiss Federal Institute of Aquatic Science and Technology, Dübendorf, Switzerland; ${ }^{3}$ School of Civil \\ Engineering and Geosciences, Newcastle University, Newcastle upon Tyne, UK; ${ }^{4}$ Department of Biological and \\ Environmental Sciences, Engineering Division, King Abdullah University of Science Technology, Thuwal, \\ Saudi Arabia and ${ }^{5}$ Center for Microbial Ecology and Technology, Ghent University, Ghent, Belgium
}

\begin{abstract}
There is a growing interest in controlling-promoting or avoiding-the invasion of microbial communities by new community members. Resource availability and community structure have been reported as determinants of invasion success. However, most invasion studies do not adhere to a coherent and consistent terminology nor always include rigorous interpretations of the processes behind invasion. Therefore, we suggest that a consistent set of definitions and a rigorous conceptual framework are needed. We define invasion in a microbial community as the establishment of an alien microbial type in a resident community and argue how simple criteria to define aliens, residents, and alien establishment can be applied for a wide variety of communities. In addition, we suggest an adoption of the community ecology framework advanced by Vellend (2010) to clarify potential determinants of invasion. This framework identifies four fundamental processes that control community dynamics: dispersal, selection, drift and diversification. While selection has received ample attention in microbial community invasion research, the three other processes are often overlooked. Here, we elaborate on the relevance of all four processes and conclude that invasion experiments should be designed to elucidate the role of dispersal, drift and diversification, in order to obtain a complete picture of invasion as a community process.
\end{abstract}

The ISME Journal (2016) 10, 2773-2779; doi:10.1038/ismej.2016.75; published online 3 May 2016

\section{Introduction}

Studies on invasion of microbial communities are gaining momentum, with a considerable increase in publications on invasion by pathogenic, beneficial or commensal microbes. A better understanding of microbial invasion could generate significant payoffs across many domains including management of human and animal diseases, development of plant growth promoting inocula and bioaugmentation in food and environmental industries. Experiments testing determinants of microbial invasion, such as resident community diversity and resource utilization, have been reported for diverse environments, including the gut of mice, the plant rhizosphere or wastewater treatment bioreactors (see Supplementary Table S1). It is, however, challenging to draw broad conclusions from these studies as the used termino-

Correspondence: BF Smets, Department of Environmental Engineering, Technical University of Denmark, Bygningstorvet 115, 2800 Kgs. Lyngby, Denmark.

E-mail: bfsm@env.dtu.dk

Received 18 November 2015; revised 6 March 2016; accepted 23 March 2016; published online 3 May 2016 logy lacks consensus, the experimental approaches are often inconsistent, and the observations are often anecdotal or even contradictory.

In plant and animal ecology, invasion has been an active research field for decades with controversies relating to its definition, boundaries and specificities (Valéry et al., 2008; Simberloff and Vitule, 2014). Controversies, such as the distinction between native and alien species, and the necessity for an invasion to have a negative impact on the resident community, are not fully resolved. Nonetheless, efforts toward a unified framework for invasion biology have been made (Blackburn et al., 2011). Similar controversies can affect the more recent field of microbial invasion-and the need to develop a general framework for microbial invasion, within which to synthesize individual observations, has been voiced before (Litchman, 2010; Mallon et al., 2015b).

Here, we propose a simple definition of invasion in a microbial community (Table 1) as the establishment of an alien microbial type in this resident community and suggest a community ecology framework to interpret this process. 
Table 1 Terminology and Definitions

\begin{tabular}{|c|c|c|}
\hline Term & Definition & Reference/Adapted from \\
\hline Microbial community & Group of potentially interacting microbial types that co-occur in space and time & Nemergut et al. (2013) \\
\hline Resident community & A specific community considered in the context of possible invasion & This study \\
\hline Alien type or invader & $\begin{array}{l}\text { A microbial type that was not part of the resident community prior to point of } \\
\text { observation }\end{array}$ & Davis et al. (2005) \\
\hline Community assembly & $\begin{array}{l}\text { The sum of all processes that shape the composition of a microbial community, } \\
\text { including dispersal, selection, drift and diversification }\end{array}$ & Vellend (2010) \\
\hline Dispersal & $\begin{array}{l}\text { Movement of organisms across space, considering immigration in and emigration } \\
\text { out of a community }\end{array}$ & Vellend (2010) \\
\hline Selection & $\begin{array}{l}\text { Changes in community composition caused by deterministic fitness differences } \\
\text { between types }\end{array}$ & Vellend (2010) \\
\hline Drift & $\begin{array}{l}\text { Changes in the relative abundance of types in a community over time caused by } \\
\text { stochastic processes }\end{array}$ & Vellend (2010) \\
\hline Diversification & $\begin{array}{l}\text { Increase in diversity of types in a community caused by generation of new genetic } \\
\text { variants }\end{array}$ & Vellend (2010) \\
\hline Invasion & Entry and establishment of an alien type in a resident community & Blackburn et al. (2011) \\
\hline Establishment & $\begin{array}{l}\text { The maintenance of an active population of an alien type in a resident community } \\
\text { for a significant period of time }\end{array}$ & This study \\
\hline
\end{tabular}

\section{Defining and detecting invasion in microbial communities}

What is an invader?

Consistent with our focus on invasion in individual microbial communities, we propose to follow the simple and inclusive definition of an invader commonly used in studies of plant or animal community invasibility, namely that any species (or-more generally-biological type) not currently part of the resident community can be considered as a potential invader (Tilman, 1997; Davis et al., 2005).

We recognize that other, more restrictive, definitions exists, especially when the focus goes beyond single communities and considers biogeography (that is, when the key interest is whether or not species expand their biogeographical range). This is the case for the invasion biology framework presented by Blackburn et al. (2011) where a potential invader should cross a significant geographical barrier. This non-nativeness implies the absence of recent ecological interactions with the resident organisms and, hence, the absence of prior co-evolution or co-adaptation. As a consequence, this type of alien organism is believed to have the potential to drastically modify the composition or functioning of the community it invades, although disagreement exists on this point (Davis et al., 2011). In this framework the invader should also demonstrate its ability to successfully disperse from the initial invasion point to other communities (Blackburn et al., 2011). A few reviews have adopted this invasion biology framework to examine microbial invasion (Litchman, 2010; Amalfitano et al., 2014), but noted that our incomplete knowledge of microbial biogeography makes it difficult to assess whether a microbial type has dispersed beyond its natural range. Other authors propose that a microbial type only qualifies as an invader if it enters 'an environment or community where it has never before existed' (Mallon et al., 2015b). This absolute, continuous, absence from the resident community is difficult to demonstrate for most communities. In summary, when the focus is on the invasion of an individual community, and because there is no indication that aliens that have dispersed out of their range are unique in their interactions with a resident community, we suggest accepting any types that are not currently present in the resident community as potential invaders.

\section{Resident community}

We define the resident community simply as any given community investigated for potential invasion. This definition is on purpose very general as it makes no assumption regarding community properties such as the existence of a temporally stable 'core' group of microbial types, of stable functional attributes, or of a long common history (for example, it includes the synthetic communities assembled shortly prior to invasion (De Roy et al., 2013; Mallon et al., 2015a)). This choice implies that invasion is not restricted to stable, closely monitored communities. However, some knowledge of the composition of the resident community is needed to establish the alien status of the invader. Cataloging all microbial types to a specific genotype can be challenging for many microbial communities, which are often immensely diverse. Therefore, the resident community can simply be defined a minima as a community that, prior to invasion, did not contain the invader (for example, see van Elsas et al., 2012 and De Roy et al., 2013). Establishing the absence of the invading type prior to invasion requires either expert knowledge of the microbial system considered or sensitive detection methods targeting the alien type directly (via growthbased, molecular or microscopic methods) or targeting a trait or function unique to the alien type. We acknowledge that absolute proof of absence is often impossible to provide outside of the specific case of synthetic communities, but demonstrating that the alien type was below the limit of detection of an 
appropriately sensitive method is generally accepted in practice (Acosta et al., 2015).

The fact that many microbes can remain dormant for long periods (Lennon and Jones, 2011) may justify a more restrictive definition of a resident community. Indeed, due to their reduced metabolic state, these microbes could be considered to have too little ecological interactions to truly be a part of the community. A dormant microbial type would then be regarded alien upon reactivation, an entry route to the resident community not traditionally considered. If such an activity-based definition has merits, it also complicates the census of the resident community.

Defining the spatial limits of a community can constitute an arduous challenge (Konopka, 2009). Although it is trivial for laboratory studies (for example, a microtiter well) or for certain systems that are clearly limited (for example, the oral cavity of a patient), other systems (for example, in soil or river) have no obvious boundaries. On the one hand, the microbial composition of these natural systems does not seem to present discontinuities as exemplified, for soils, by the progressive decrease with distance of the genetic similarity of the members of a free-living bacterial species (Vos and Velicer, 2008). On the other hand, significant variations of antibiotic inhibitory interactions among Streptomycete communities on an area of $1 \mathrm{~m}^{2}$ have been observed, which suggest distinct selective environments among these locations (Davelos et al., 2004). In this case, community boundaries could be defined through similar antibiotic-inhibition abilities. Diffuse boundaries obviously challenge the applicability of the concept of community invasion in many natural ecosystems as it does for other community ecology concepts (Konopka, 2009), but attempts to define spatial limits of a resident community can be made even in highly complex natural systems.

\section{Establishment}

Although the first event in community invasion-the entry of an alien microbial type-is self-explanatory, defining and accurately measuring the next stepestablishment-is more difficult. In general ecology, establishment is defined as the persistence of the alien type as a self-sustaining population in the new environment, owing to exploitation of its resources (Davis et al., 2005). For many microbes, a single cell theoretically suffices to develop a population, but we propose the maintenance of a metabolically active population of invaders for a significant period of time, as a better operative criterion. Qualifying further the 'significant' period of time needs to be based on the characteristics of the resident community, because communities can differ significantly in biomass turnover rate and temporal variability. A time horizon for establishment can be based on the average renewal time $\left(T_{R}\right)$ of the biomass in the community. This can be calculated as $T_{R}=\mathrm{N} /\left(\mathrm{Y} \times F_{S}\right)$; where $N$ (biomass) is the amount of biomass of the resident community, $F_{S}$ is the flux of substrate to the community (mass of substrate per unit time), and Y, the 'effective' yield of the community (biomass per mass substrate). We suggest monitoring the community over a few average renewal times before concluding on the alien's establishment. It is also relevant to include several iterations of any known cycles the habitat is exposed to (for example, seasonal cycles for natural communities, operational cycles for engineered systems) before deeming the invasion successful. Ensuring that the alien maintains an active population may be warranted if suspicion exists that the alien might only persist in dormant form (for example, spores) or even as nucleic acid remains, which could still be detected by PCR, for example. This demonstration is not required if the population dynamics of the alien indicates local growth, for example, by reaching larger abundance than upon entry (increase from rarity), an unlikely occurrence in many invasion experiments where the alien type is introduced at very high abundance.

\section{Invasion consequences}

A debated criterion in defining invasion pertains to its consequences. In accordance with Blackburn et al. (2011), we propose omitting the requirement that the establishment of the invader negatively impacts the community. This omission is controversial due to the negative connotation of the word 'invasion', but we argue that 'negative impact' is a vague criterion. Indeed, 'impact' can be measured in multiple ways on myriads of different community attributes (abundance of resident populations, community richness, community functional attributes, and so on) that may not directly relate to the invader, because the establishment of an invader can have both positive and negative consequences on different community members (Valéry et al., 2008).

\section{Consistency between reported studies and proposed definitions?}

Although there are many applied studies that may be relevant to the framework presented here, including biofilm colonization, bioaugmentation and competition studies, we focused our evaluation of the literature on studies specifically using the term 'invasion' (Supplementary Table S1). These studies, however, do not use explicit definitions or measurement criteria. For example, the concept of a resident community is often approached loosely, particularly when synthetic assembled communities are added together with the 'invader'. In these cases, nothing, from an ecological perspective, distinguishes the 'resident' community members from the 'invader' because none have had a prior common existence. In many studies, proper controls proving the absence of the invader from the resident community are either missing or not reported. Finally, many studies declare successful establishment when persistence is assessed over a short, unjustified time period. 
Microbial invasion in a community ecology perspective

We can categorize the invasion literature into two classes, depending on whether the focus is on the invader or on the resident community. Invader-centric research focuses on the invader's traits (Hambright et al., 2014) and, for example, compares the invasiveness of different alien types (Ma et al., 2015). The largest share of the literature, however, is 'resident community-centric' and thus evaluates community invasibility (Supplementary Table S1). Often, the authors create an array of communities with variation in an attribute (for example, richness, evenness, genomic dissimilarity) by manipulating natural communities (for example decreasing diversity via dilutions and stress perturbations) or by assembling synthetic communities and evaluate the importance of that attribute in invasibility. Synthetic communities, assemblages of a limited number of pure strains (De Roy et al., 2013) sometimes all belonging to the same species (Hodgson et al., 2002; Eisenhauer et al., 2013), are very attractive because they afford great control on experimental design (De Roy et al., 2014). However, it is unknown to what extent these communities are suitable models for natural communities where more complex ecological interactions likely exist. Thus, the success of invasion in synthetic communities might depend on different factors compared with natural communities, where a longer history of ecological interactions has shaped the community composition.

The reviewed studies on invasion do not adhere to a systematic ecological framework. In most experimental studies that have a community ecology emphasis, the focus is on the competition for electron donors and/or acceptors as well as micronutrients. However, this common focus is sometimes obscured by the use of a variety of terms across papers (for example, niche overlap, niche dimensionality, resource utilization). Therefore, we propose that adopting the framework of community ecology developed by Vellend (2010) can help clarify the potential determinants of invasion. This framework identifies four fundamental processes that control community dynamics: (1) selection, (2) drift, (3) diversification and (4) dispersal.

Dispersal includes both immigration and emigration. An alien type will have more opportunities to immigrate into a community when dispersal to the resident community is strong. Dispersal is not considered in the majority of microbial-invasion studies conducted in closed-batch systems (Supplementary Table S1), but may be critical to invasion success. The dispersal rate, defined as the number of entering cells per unit of time, largely depends on the environment. Lindström and Langenheder (2012) reviewed the regional factors that can influence the dispersal rate in a given environment. They suggest that dispersal limitation has a bigger role in terrestrial environments compared with aquatic environments. As dispersal is mainly passive for bacteria, at least on macroscopic spatial scales (for example dispersal via air, aerosols or with water flow in soil environment), it can be modeled using mechanistic aerodynamic and hydrodynamic models similar to those used for plant seed and zooplankton dispersal (Michels et al., 2001; Kuparinen et al., 2007). The abundance of an alien type population entering the resident community can be quantified as the product of its dispersal rate by the duration of dispersal. If this abundance is low, the probability of establishment can be reduced (Blackburn et al., 2015) because a few immigrants are more subject to stochastic events that may lead to their extinction. This assertion has been confirmed for macro-organisms, but is rarely addressed for microbial communities, although a recent study on algal invasion provides supportive evidence that the quantity of invader is key determinant of invasion success (Acosta et al., 2015). In fact, in most of the literature we reviewed, the alien type is added in high numbers, during a single immigration event (Supplementary Table S1).

After alien entry, selection and/or drift are the main processes determining its establishment. Selection pressure on the invader can be positive or negative depending on the resident community's composition, its dynamics and the environmental conditions. This can be conceptualized by considering the potential niche overlap between the alien type and the resident community. In extreme cases, the alien can fill an empty niche because it possesses metabolic traits absent from the community (transiently or long-term) and can thus easily establish (the case of bioaugmentation). More commonly, the alien type is required to outcompete at least one member of the resident community in order to establish itself. This can occur if the alien possesses traits that allow it to displace a resident from the same niche. The role of selectionand specifically of competition for resources-as a determinant of invasion has been the subject of several studies on microbial community invasion (Supplementary Table S1). Richness (and/or genomic dissimilarity) of the resident community is often used as a proxy for its ability to compete with the alien type (Dunstan and Johnson, 2006; Jousset et al., 2011), because more diverse communities have higher probability to host resident types that are competitively superior to the alien either as individuals or as interacting types (Figure 1a). This assumption is sometimes supported experimentally by measuring the resource-utilization pattern or the productivity of the resident communities (as a whole or as individual members) and of the alien type (for example, see Hodgson et al., 2002). Indeed, more diverse resident communities are usually less prone to the establishment of an invader. In a similar vein, invasion success increases with phylogenetic distance between the alien and the resident species in synthetic communities of a few bacterial strains (Jiang et al., 2010). The importance of the resident community composition implies that the timing of the alien entry relative to the prior history of the resident community may also be determinant in the invasion success. 

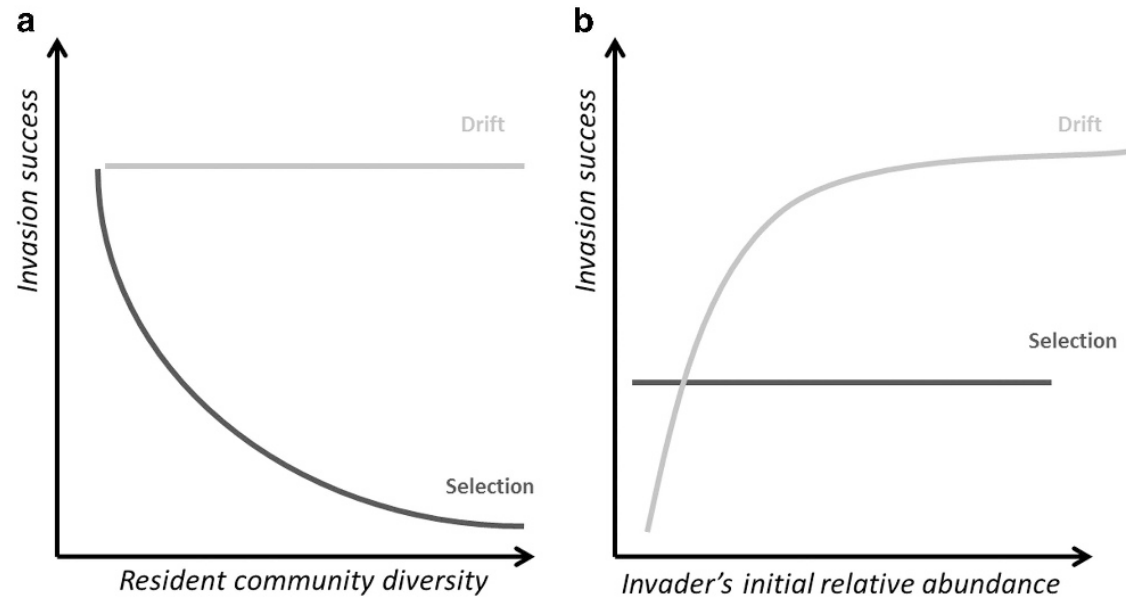

Figure 1 Hypothetical relation between average invasion success and (a) resident community diversity, and (b) initial relative abundance of the invader, in communities where either competition (selection) or drift govern the community assembly. In a, the exponential curve is illustrative; the curve can be of any monotonic form, with the rate of decline depending on the distribution of the competitive abilities of the resident microbes relative to that of the alien. In $\mathbf{b}$, the curve associated with drift highlights the fact that, in a drift-only situation, invasion success increases rapidly with the size of the invading population. For example, simulating drift with the untb package (Hankin, 2007) shows that if an alien enters at $1 \%$ relative abundance or more in a community with a death and birth rate of $5 \%$ per generation, invasion success (persistence of at least one alien cell) after 5 biomass turnover times is almost certain.

Communities that have had less time to assemble are likely to contain fewer types with broader niches and lower competitive abilities than communities that have 'matured' longer (Shea and Chesson, 2002). Invasion success would then be higher in less mature communities.

Selection in a community can be markedly affected when the community is subjected to ecological disturbance (Shade et al., 2012; Ma et al., 2015). Disturbances tend to decrease community density and diversity, potentially increasing the available niche space - in other words temporarily decreasing competition. The invader establishment then depends on its ability to cover the available niche space of the disturbed community (Liu et al., 2012; De Roy et al., 2013).

Neutral drift refers to a process where community structure changes stochastically through demographic processes of 'birth' (or division) and death. If all community members have equal fitness, then drift is important: the establishment of the alien type is then random, depending mainly on its relative and absolute abundance (Figure 1b). Under pure drift, the long-term probability of invader disappearance is one minus its initial frequency, and the rate of disappearance is negatively related to community size (Vellend, 2010). Although it cannot be easily quantified, drift is always present in biological communities but this process is especially important in communities of low abundance, because discrete division/death events have stronger consequences. Therefore, a disturbance that durably decreases the size of the community would increase the role of drift.

Current invasion research focuses predominantly on the correlation between invasion success and resident community diversity. If these studies have contributed to our understanding of how communities dominated by selection are responding to invasion, we emphasize that further considerations are necessary. The dynamics of microbial communities are usually governed by more than one process and, as we indicated above, in communities where drift is stronger than selection, resident community diversity will not have a critical role in invasion success. Instead, the initial relative and absolute abundance of the invader should be a key determinant of establishment (Figure 1).

Finally, communities can increase their diversity through the acquisition of new genotypes that emerge from ancestral genotypes due to diversification. Diversification can occur in the invader population or in the resident community and is thought to be positively related to population size. Some bacteria can diversify rapidly (within a few days) when introduced at high abundance in a habitat, even in the presence of a resident community (Gómez and Buckling, 2013). Diversification can increase the chances for an alien type to establish if some of the mutations are adaptive (Tayeh et al., 2015). Conversely, diversification of the resident community members might produce insurance effects and increase community resistance to invasion.

\section{Recommendation for future research}

We proposed a definition of invasion where every microbial type not present at a particular time in a community is a potential invader. This is appealing because this framework is consistent with accepted ecological research terminology and is easily and coherently applicable to any microbial community. It not only clearly highlights the fundamental ecological similarity between seemingly disparate concepts such as bioaugmentation, pathogen suppressive communities or microbiome enhancement, but it also indicates that community invasion should be 
considered as a 'normal' event in community assembly and is commonplace in most communities. However, more restrictive definitions might be needed once we know more about the stability of microbial communities. For example such future definitions may exclude conditionally rare types from the scope of invasion because of their frequent transient occurrence within communities. In addition, the resident community may need to be defined more clearly as a community with historical interactions among its members and with its environment.

By presenting invasion research in a community ecology framework, it is apparent that some processes have received more attention than others. A selection focus has overwhelmed invasion research, while other processes shaping community dynamics are rarely considered. For example, in batch experiments with sterile feeds, dispersal in the community is often completely interrupted, except from the introduction of the alien type. Therefore, the impact of the dynamics of the resident community on invasion is neglected. Similarly, the absence or the constant supply of nutrients to the community during an invasion experiment might not simulate the native conditions of the community correctly. In fact, when nutrients are supplied in a dynamic manner, the outcome of the invasion can change (Liu et al., 2012; Mallon et al., 2015a). Overall, we suggest designing experiments that elucidate the role of dispersal, drift, and diversification, because these processes have received less attention. In this way, a complete picture of invasion as a community process can be obtained.

The discussed community ecological framework, combined with appropriate experimental conditions and measures of invasion, can be used to posit and test hypotheses on invasion that consider alien type and resident community in one coherent framework. We emphasize that invasion should be considered as a part of 'normal' community assembly and not only seen as something with negative consequences. The applications of such framework range from controlling environmental processes like water treatment to human microbiome improvement. We believe that adopting a coherent conceptional framework efforts will progress the study of microbial invasion.

\section{Conflict of Interest}

The authors declare no conflict of interest.

\section{Acknowledgements}

This perspective is an outcome of discourses during the crossinstitutional projects MERMAID (an initial training network funded by the People Programme-Marie Skłodowska-Curie Actions- of the European Union's Seventh Framework Programme FP7/2007-2013/ under REA grant agreement no. 607492) and the Inter-University Attraction Pole (IUAP) project $\mu$-manager funded by the Belgian Science Policy (BELSPO, 305 P7/25). We also acknowledge support from the King Abdullah University of Science and Technology.

\section{References}

Acosta F, Zamor RM, Najar FZ, Roe BA, Hambright KD. (2015). Dynamics of an experimental microbial invasion. Proc Natl Acad Sci USA 112: 11594-11599.

Amalfitano S, Coci M, Corno G, Luna GM. (2014). A microbial perspective on biological invasions in aquatic ecosystems. Hydrobiologia 746: 13-22.

Blackburn TM, Lockwood JL, Cassey P. (2015). The influence of numbers on invasion success. Mol Ecol 24: 1942-1953.

Blackburn TM, Pyšek P, Bacher S, Carlton JT, Duncan RP, Jarošík V et al. (2011). A proposed unified framework for biological invasions. Trends Ecol Evol 26: 333-339.

Davelos AL, Kinkel LL, Samac DA. (2004). Spatial variation in frequency and intensity of antibiotic interactions among Streptomycetes from prairie soil. Appl Environ Microbiol 70: 1051-1058.

Davis MA, Chew MK, Hobbs RJ, Lugo AE, Ewel JJ, Vermeij GJ et al. (2011). Don't judge species on their origins. Nature 474: 153-154.

Davis MA, Thompson K, Grime JP. (2005). Invasibility: the local mechanism driving community assembly and species diversity. Ecography 28: 696-704.

De Roy K, Marzorati M, Negroni A, Thas O, Balloi A, Fava $\mathrm{F}$ et al. (2013). Environmental conditions and community evenness determine the outcome of biological invasion. Nat Commun 4: 1383.

De Roy K, Marzorati M, Van den Abbeele P, Van de Wiele T, Boon N. (2014). Synthetic microbial ecosystems: an exciting tool to understand and apply microbial communities. Environ Microbiol 16: 1472-1481.

Dunstan PK, Johnson CR. (2006). Linking richness, community variability, and invasion resistance with patch size. Ecology 87: 2842-2850.

Eisenhauer N, Schulz W, Scheu S, Jousset A. (2013). Niche dimensionality links biodiversity and invasibility of microbial communities. Funct Ecol 27: 282-288.

Gómez P, Buckling A. (2013). Real-time microbial adaptive diversification in soil. Ecol Lett 16: 650-655.

Hambright DK, Beyer JE, Easton JD, Zamor RM, Easton AC, Hallidayschult TC. (2014). The niche of an invasive marine microbe in a subtropical freshwater impoundment. ISME J 9: 1-9.

Hankin RKS. (2007). Introducing untb, an R package for simulating ecological drift under the unified neutral theory of biodiversity. J Stat Softw 22: 1-15.

Hodgson DJ, Rainey PB, Buckling A. (2002). Mechanisms linking diversity, productivity and invasibility in experimental bacterial communities. Proc R Soc B Biol Sci 269: 2277-2283.

Jiang L, Tan J, Pu Z. (2010). An experimental test of Darwin's naturalization hypothesis. Am Nat 175: 415-423.

Jousset A, Schulz W, Scheu S, Eisenhauer N. (2011). Intraspecific genotypic richness and relatedness predict the invasibility of microbial communities. ISME J 5: 1108-1114.

Konopka A. (2009). What is microbial community ecology? ISME J 3: 1223-1230.

Kuparinen A, Markkanen T, Riikonen H, Vesala T. (2007). Modeling air-mediated dispersal of spores, pollen and seeds in forested areas. Ecol Modell 208: 177-188.

Lennon JT, Jones SE. (2011). Microbial seed banks: the ecological and evolutionary implications of dormancy. Nat Rev Microbiol 9: 119-130. 
Lindström ES, Langenheder S. (2012). Local and regional factors influencing bacterial community assembly. Environ Microbiol Rep 4: 1-9.

Litchman E. (2010). Invisible invaders: non-pathogenic invasive microbes in aquatic and terrestrial ecosystems. Ecol Lett 13: 1560-1572.

Liu M, Bjørnlund L, Rønn R, Christensen S, Ekelund F. (2012). Disturbance promotes non-indigenous bacterial invasion in soil microcosms: analysis of the roles of resource availability and community structure. PLoS One 7: e45306.

Ma C, Liu M, Wang H, Chen C, Fan W, Griffiths B et al. (2015). Resource utilization capability of bacteria predicts their invasion potential in soil. Soil Biol Biochem 81: 287-290.

Mallon CA, Poly F, Le Roux X, Marring I, van Elsas JD, Salles JF. (2015a). Resource pulses can alleviate the biodiversity - invasion relationship in soil microbial communities. Ecology 96: 915-926.

Mallon CA, van Elsas JD, Salles JF. (2015b). Microbial invasions: the process, patterns, and mechanisms. Trends Microbiol 23: 719-729.

Michels E, Cottenie K, Neys L, De Gelas K, Coppin P, De Meester L. (2001). Geographical and genetic distances among zooplankton populations in a set of interconnected ponds: a plea for using GIS modelling of the effective geographical distance. Mol Ecol 10: 1929-1938.
Shade A, Peter H, Allison SD, Baho DL, Berga M, Bürgmann H et al. (2012). Fundamentals of microbial community resistance and resilience. Front Microbiol 3: 1-19.

Shea K, Chesson P. (2002). Community ecology theory as a framework for biological invasions. Trends Ecol Evol 17: $170-176$.

Simberloff D, Vitule JRS. (2014). A call for an end to calls for the end of invasion biology. Oikos 123: 408-413.

Tayeh A, Hufbauer RA, Estoup A, Ravigné V, Frachon L, Facon B. (2015). Biological invasion and biological control select for different life histories. Nat Commun 6: 7268 .

Tilman D. (1997). Community invasibility, recruitment limitation, and grassland biodiversity. Ecology 78: 81-92.

Valéry L, Fritz H, Lefeuvre J-C, Simberloff D. (2008). In search of a real definition of the biological invasion phenomenon itself. Biol Invasions 10: 1345-1351.

van Elsas JD, Chiurazzi M, Mallon CA, Elhottova D, Chiurazzi M, Mallon CA et al. (2012). Microbial diversity determines the invasion of soil by a bacterial pathogen. Proc Natl Acad Sci USA 109: 1159-1164.

Vellend M. (2010). Conceptual synthesis in community ecology. $Q$ Rev Biol 85: 183-206.

Vos M, Velicer GJ. (2008). Isolation by distance in the spore-forming soil bacterium Myxococcus xanthus. Curr Biol 18: 386-391.

Supplementary Information accompanies this paper on The ISME Journal website (http://www.nature.com/ismej) 